\title{
Functional gastroduodenal disorders
}

\author{
N J Talley, V Stanghellini, R C Heading, K L Koch, J R Malagelada, G N J Tytgat
}

Chair, Committee on Functional

Gastroduodenal

Disorders,

Multinational Working

Teams to Develop

Diagnostic Criteria for

Functional

Gastrointestinal

Disorders (Rome II),

Department of

Medicine, University

of Sydney,

Penrith, Australia

N J Talley

Co-Chair, Committee on Functional

Gastroduodenal

Disorders,

Multinational Working

Teams to Develop

Diagnostic Criteria for

Functional

Gastrointestinal

Disorders (Rome II),

Department of

Internal Medicine and

Gastroenterology,

Bologna, Italy

V Stanghellini

University of

Edinburgh,

Edinburgh, Scotland,

UK

R C Heading

Milton S Hershey

Medical Center,

Hershey, PA, USA

K L Koch

Digestive System

Research Unit,

Hospital General Vall

D'Hebron,

Barcelona, Spain

J R Malagelada

Division of

Gastroenterology and

Hepatology, Academic

Medical Center,

Amsterdam, The

Netherlands

G N J Tytgat

Correspondence to: Nicholas J Talley, $\mathrm{MD}$

Professor of Medicine,

University of Sydney,

Clinical Sciences Building,

Nepean Hospital, PO Box

63, Penrith NSW 2751,

Australia.
Abstract

While widely used in research, the 1991

Rome criteria for the gastroduodenal disorders, especially symptom subgroups in dyspepsia, remain contentious. After a comprehensive literature search, a consensus-based approach was applied, supplemented by input from international experts who reviewed the report. Three functional gastroduodenal disorders are defined. Functional dyspepsia is persistent or recurrent pain or discomfort centered in the upper abdomen; evidence of organic disease likely to explain the symptoms is absent, including at upper endoscopy. Discomfort refers to a subjective, negative feeling that may be characterized by or associated with a number of non-painful symptoms including upper abdominal fullness, early satiety, bloating, or nausea. A dyspepsia subgroup classification is proposed for research purposes, based on the predominant (most bothersome) symptom: (a) ulcer-like dyspepsia when pain (from mild to severe) is the predominant symptom, and (b) dysmotility-like dyspepsia when discomfort (not pain) is the predominant symptom. This classification is supported by recent evidence suggesting that predominant symptoms, but not symptom clusters, identify subgroups with distinct underlying pathophysiological disturbances and responses to treatment. Aerophagia is an unusual complaint characterized by air swallowing that is objectively observed and troublesome repetitive belching. Functional vomiting refers to frequent episodes of recurrent vomiting that is not self-induced nor medication induced, and occurs in the absence of eating disorders, major psychiatric diseases, abnormalities in the gut or central nervous system, or metabolic diseases that can explain the symptom. The current classification requires careful validation but the criteria should be of value in future research.

(Gut 1999;45(Suppl II):II37-II42)

Keywords: dyspepsia; functional dyspepsia; aerophagia; psychogenic vomiting; Rome II

Based on the consensus opinion of an international panel of clinical investigators who reviewed the available evidence, a classification of the functional gastroduodenal disorders (category B) into functional dyspepsia (category B1), aerophagia (category B2), and functional vomiting (category B3) is recommended (table 1). In addition, symptom subgroups for functional dyspepsia are pro-
Table 1 Functional gastrointestinal disorders

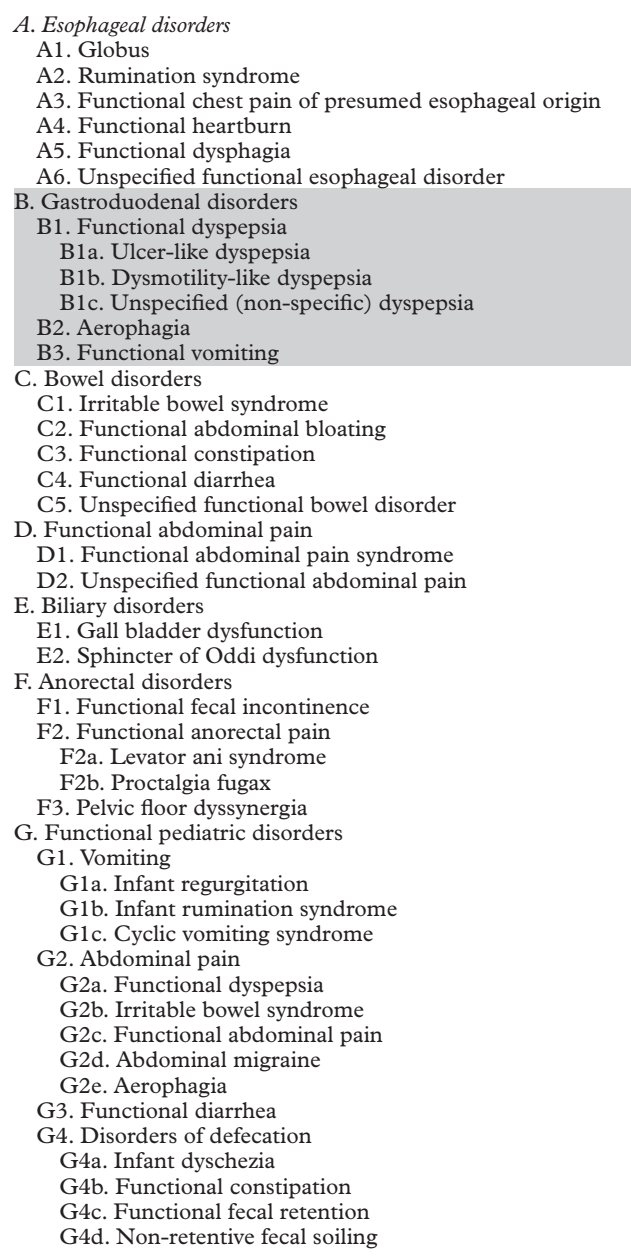

posed, namely ulcer-like dyspepsia (B1a) and dysmotility-like dyspepsia (B1b), based on patient ranking of the most bothersome complaint. The evidence in support of this classification is summarized here, and an approach to management reviewed.

\section{B1. Functional dyspepsia}

DEFINITION

Although many definitions have been proposed, the committee, following a review of the literature, endorsed the original Rome definition. ${ }^{1}$ Hence, dyspepsia refers to pain or discomfort centered in the upper abdomen.

Centered implies that the pain or discomfort is mainly in or around the midline. Pain in the right or left hypochondrium is not considered to be representative of dyspepsia. Discomfort

Abbreviations used in this paper: GERD, gastro-esophageal reflux disease; IBS, irritable bowel syndrome; EGG, electrogastrography. 
Table 2 The spectrum of dyspepsia symptoms and recommended definitions

\begin{tabular}{|c|c|}
\hline Symptom & Definition \\
\hline Pain centered in the upper abdomen & $\begin{array}{l}\text { Pain refers to a subjective, unpleasant sensation; some } \\
\text { patients may feel that tissue damage is occurring. Other } \\
\text { symptoms may be extremely bothersome without being } \\
\text { interpreted by the patient as pain. By questioning the } \\
\text { patient, pain should be distinguished from discomfort. }\end{array}$ \\
\hline Discomfort centered in the upper abdomen & $\begin{array}{l}\text { A subjective, unpleasant sensation or feeling that is not } \\
\text { interpreted as pain according to the patient and which, if } \\
\text { fully assessed, can include any of the symptoms below. }\end{array}$ \\
\hline Early satiety & $\begin{array}{l}\text { A feeling that the stomach is overfilled soon after starting to } \\
\text { eat, out of proportion to the size of the meal being eaten, } \\
\text { so that the meal cannot be finished. }\end{array}$ \\
\hline Fullness & $\begin{array}{l}\text { An unpleasant sensation like the persistence of food in the } \\
\text { stomach; this may or may not occur postprandially (slow } \\
\text { digestion). }\end{array}$ \\
\hline Bloating in the upper abdomen & $\begin{array}{l}\text { A tightness located in the upper abdomen; it should be } \\
\text { distinguished from visible abdominal distension }\end{array}$ \\
\hline Nausea & Queasiness or sick sensation; a feeling of the need to vomit \\
\hline
\end{tabular}

refers to a subjective, negative feeling that the patient does not interpret as pain and which, if fully assessed, can include a number of specific symptoms. Discomfort may be characterized by or associated with upper abdominal fullness, early satiety, bloating, or nausea; these symptoms typically are accompanied by a component of upper abdominal distress (table 2).

Duration is not specified as part of the definition because patients may present immediately following the onset of symptoms or may wait months or years before being evaluated. In research studies, investigators may opt for a specified duration of symptoms to define dyspepsia (e.g., two, four, or 12 weeks), and this should depend on the study setting and objectives so as to improve homogeneity of the patients being studied. The painful or uncomfortable symptoms may be intermittent or continuous, and may or may not be related to meals.

There is growing consensus based on 24 hour esophageal $\mathrm{pH}$ testing that patients with a history of typical heartburn, when this is a dominant complaint, have symptomatic gastroesophageal reflux disease (GERD), until proved otherwise. ${ }^{2}$ There is reasonably good evidence that predominant heartburn is sensitive and specific for GERD, and hence the positive predictive value for heartburn is high in countries where GERD is a common disease. ${ }^{3}$ How many patients with burning epigastric pain alone truly have GERD is unknown.

Uninvestigated versus investigated dyspepsia

It is important to distinguish the patient who presents with dyspepsia that has not been investigated (uninvestigated dyspepsia) from patients with a diagnostic label after investigation, with either a structural diagnosis (such as peptic ulcer or GERD), or functional dyspepsia (fig 1).

\section{Causes of dyspepsia}

From an etiological viewpoint, patients with dyspepsia can be subdivided into three main categories ${ }^{1}$ :

(1) those with an identified cause for the symptoms (e.g., chronic peptic ulcer disease, gastroesophageal reflux disease with or without esophagitis, malignancy, pancreatico-biliary disease, and medications);

(2) those with an identifiable pathophysiological or microbiologic abnormality of uncertain clinical relevance (e.g., Helicobacter pylori gastritis, histologic duodenitis, gallstones, visceral hypersensitivity, gastroduodenal dysmotility); and

(3) those with no identifiable explanation for the symptoms.

It is those patients who have no definite structural or biochemical explanation for their symptoms (i.e., categories 2 and 3 ) that are considered to have functional dyspepsia. The term non-ulcer dyspepsia remains in popular usage, but is not recommended here because some patients with functional dyspepsia will have symptoms not at all like an ulcer, and peptic ulcer is not the only disease of exclusion in patients with dyspepsia. ${ }^{4}$

\section{DIAGNOSTIC CRITERIA}

Functional dyspepsia

Symptom patterns alone are unable to adequately discriminate organic from functional dyspepsia. ${ }^{4}$ Patients need to have been investigated to rule out relevant organic disease. Functional dyspepsia therefore remains a diagnosis of exclusion. There is agreement that symptoms should have run a chronic course before a patient is labeled as having functional dyspepsia. It is therefore recommended that functional dyspepsia be defined as follows:

At least 12 weeks, which need not be consecutive, within the preceding 12 months of:

(1) Persistent or recurrent dyspepsia (pain or discomfort centered in the upper abdomen); and

(2) No evidence of organic disease (including at upper endoscopy) that is likely to explain the symptoms; and

(3) No evidence that dyspepsia is exclusively relieved by defecation or associated with the onset of a change in stool frequency or stool form (i.e., not irritable bowel). 


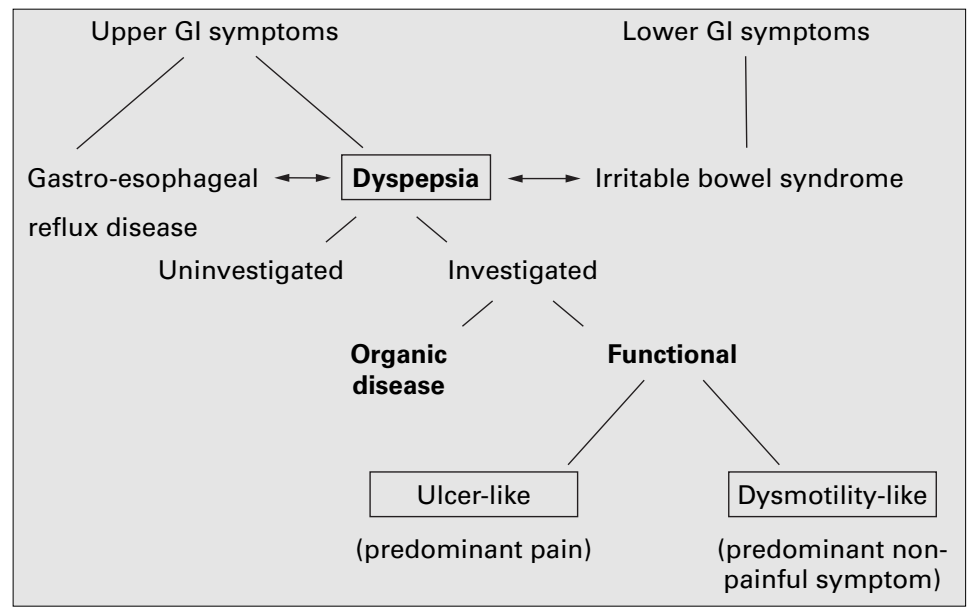

Figure 1 Relationship between dyspepsia and functional dyspepsia. GI, gastrointestinal.

The minimum diagnostic workup required to sustain a diagnosis of functional dyspepsia in the research context is a careful history and physical examination, and an upper endoscopy during a symptomatic period off antisecretory therapy. In the research setting, additional investigations depend on the research question.

Patients with a past history of documented chronic peptic ulcer disease should generally not be classified as having functional dyspepsia. However, patients who have had ulcer disease cured by $H$ pylori eradication may remain symptomatic $^{6}$; a subset of these patients probably has functional dyspepsia although this remains poorly documented.

\section{Functional dyspepsia subgroups}

The concept of subdividing dyspepsia into subsets based on distinctive symptom patterns continues to be controversial, but has become entrenched in clinical practice. ${ }^{7}$ Four working teams have independently proposed that functional dyspepsia be subdivided into symptom subgroups, ${ }^{18-10}$ but the definitions of the subgroups have not been uniform. In contrast to other groups, the previous Rome criteria proposed not to include the reflux-like subgroup in functional dyspepsia as these patients have GERD until proved otherwise. ${ }^{1}$ Two main hypothetical subgroups were identified in all the reports: ulcer-like and dysmotilitylike dyspepsia, characterized respectively by aspects of pain, or by symptoms distinct from pain and suggestive of impaired gastroduodenal motility. Two working team reports introduced numeric limitations by requiring the concomitant presence of at least two ${ }^{9}$ or three $^{1}$ specific symptoms to be included in a subgroup, thus arbitrarily introducing the concept of symptom clusters.

Epidemiologic, pathophysiologic, and clinical studies over the past five years, have demonstrated that the symptom cluster classification is of no clinical utility. ${ }^{41}$ The dyspepsia subgroup classification proposed here is therefore based not on symptom clusters but on the predominant (or most bothersome) single symptom identified by the patient as defined below:

\section{B1a. Ulcer-like dyspepsia}

Pain centered in the upper abdomen is the predominant (most bothersome) symptom.

\section{B1b. Dysmotility-like dyspepsia}

An unpleasant or troublesome non-painful sensation (discomfort) centered in the upper abdomen is the predominant symptom; this sensation may be characterized by or associated with upper abdominal fullness, early satiety, bloating, or nausea.

Recent studies support the existence of such subgroups, as defined by the presence of a predominant (or most bothersome) single symptom identified by the patient. Stanghellini et al showed that female gender, postprandial fullness severe enough to influence usual activities, and vomiting severe enough to change usual activities were independently associated with delayed gastric emptying, but clusters of symptoms were not associated. ${ }^{12}$ Tack et al observed that early satiety was specifically linked to impaired gastric accommodation. ${ }^{13}$ The response to proton pump inhibitor therapy in two large trials was linked to subgrouping based on the most bothersome symptom (those with epigastric pain but not discomfort had a statistically significant response to omeprazole over placebo), but this was not observed using the approach of symptom clusters. ${ }^{14}$ Although not all studies agree, ${ }^{15}$ the proposed subgroups represent a reasonable working hypothesis, although they must be subjected to further careful validation.

\section{B1c. Unspecified (non-specific) dyspepsia}

Symptomatic patients whose symptoms do not fulfill the criteria for ulcer-like or dysmotility-like dyspepsia.

\section{Overlap syndromes}

Heartburn - Patients in whom heartburn (i.e., burning retrosternal pain) is the predominant symptom are excluded from dyspepsia by definition and almost invariably have GERD. ${ }^{24}$ However, patients with functional dyspepsia very often have heartburn as an additional symptom subordinate to their abdominal pain or discomfort. In many of these cases the heartburn is so minor or infrequent that it cannot be considered abnormal. A minority of others with frequent heartburn, if fully investigated, are found to have pathological acid reflux on ambulatory esophageal $\mathrm{pH}$ monitoring; they have GERD and their inclusion in functional dyspepsia is then recognized to be mistaken. ${ }^{16}$

Irritable bowel syndrome-Dyspepsia occurs commonly in patients with symptoms otherwise compatible with irritable bowel syndrome (IBS). ${ }^{17}$ Despite this overlap, most patients 
with functional dyspepsia do not have significant bowel symptoms if strict definitions are applied, ${ }^{17}$ and dyspepsia is likely to comprise a distinct syndrome based on factor analysis studies. ${ }^{18}$ However, it is possible for individuals to have both functional dyspepsia and IBS, or have upper abdominal pain or discomfort exclusively related to IBS. Therefore, if upper abdominal pain or discomfort is exclusively relieved by defecation and/or is associated with a change in bowel pattern, IBS is the diagnosis by definition. On the other hand, if there is pain or discomfort in the upper abdomen that is unrelated to bowel pattern and there is other pain or discomfort that is related to bowel pattern, then functional dyspepsia and IBS can be considered to coexist.

\section{RATIONALE FOR CHANGES IN DIAGNOSTIC}

CRITERIA

The definition of functional dyspepsia is essentially unchanged. It was, however, not felt to be useful to restrict the definition arbitrarily to a set number of days over a three month period in research or practice, and thus this part was deleted. Although patients with predominant heartburn are excluded from the functional dyspepsia category, patients with IBS are not in the new classification. The definitions of the dyspepsia subgroups have been altered based on clinical, epidemiological, and therapeutic evidence that the original concept of symptom clusters are of no value. In contrast, a classification applying the predominant symptom may identify distinct groupings. ${ }^{12-14}$ This approach, however, still requires careful validation.

\section{CLINICAL EVALUATION}

The management of the patient with uninvestigated dyspepsia should not be confused with the approach to the patient with documented functional dyspepsia.

\section{Uninvestigated dyspepsia}

The current best test for excluding structural causes of dyspepsia is upper endoscopy. ${ }^{4}$ However, there is growing consensus that $H$ pylori testing is a useful approach for managing new patients. ${ }^{419}$ Among those with uninvestigated dyspepsia who are $H$ pylori positive, a substantial number will have peptic ulcer disease. ${ }^{20}$ Endoscopy can now generally be reserved for patients who present with symptoms for the first time in older age or who have alarm features such as weight loss, vomiting, dysphagia, or bleeding that strongly suggest structural disease may be present. ${ }^{4}$

\section{Functional dyspepsia}

Performance of an upper endoscopy during a symptomatic period off acid suppressant therapy is essential to identify functional dyspepsia appropriately by excluding other important structural diseases. Ultrasonography is not recommended as a routine clinical test, as in outpatient studies most patients have no detectable abnormality in the absence of symptoms or biochemical tests suggestive of biliary tract or pancreatic disease. ${ }^{21}$ Barium radiography of the small bowel is useful if there is any suspicion of mechanical obstruction.

A gastric emptying study (e.g., scintigraphy) is not currently recommended as a routine clinical test. Indeed, there is no convincing evidence that prokinetics are more efficacious in those patients with functional dyspepsia who have delayed versus normal gastric emptying, ${ }^{22}$ although conflicting results have been published. ${ }^{23}$ In patients with resistant symptoms, a gastric emptying test may be considered. If gastroparesis is found on a gastric emptying study, then specific causes of gastroparesis need to be excluded, and in particular, intestinal obstruction, diabetes mellitus, connective tissue disease, and drugs. Gastroduodenal manometry and electrogastrography (EGG) provide objective evidence of neuromuscular dysfunction in selected patients, and their role in clinical medicine is evolving at this time.

Patients with chronic or severe symptoms may benefit from an appropriate psychiatric history being taken to exclude depression, somatoform disorder, and an eating disorder.

\section{TREATMENT}

The range of therapies prescribed for functional dyspepsia reflects the uncertain pathogenesis and the lack of satisfactory treatment options. Management is further confounded by high placebo response rates: between 20 and $60 \%$ of patients with functional dyspepsia have symptom improvement on placebo. ${ }^{24} 25$ Drug therapy is not always required: a proportion of patients will respond satisfactorily to reassurance and explanation.

Anecdotally, dyspeptic symptoms may be lessened by avoiding offending foods, a high fat diet, coffee, alcohol, and cigarette smoking. If early satiety, postprandial bloating, or nausea is dominant, taking six small low fat meals per day may help decrease the intensity of the symptoms. However, none of these interventions is of established value.

Although antacids are commonly taken by patients with dyspepsia, double blind controlled trials have shown they are not superior to placebo in functional dyspepsia. ${ }^{26} \mathrm{H}_{2}$ receptor antagonists are widely prescribed for dyspeptic patients, but double blind controlled trials have reported mixed results ${ }^{24}$; other data suggest that responders to $\mathrm{H}_{2}$ blockers may be restricted to a subset with unrecognized GERD. ${ }^{27}$ A meta-analysis has suggested a therapeutic gain of approximately $20 \%$ over placebo, which is comparable with the response obtained with prokinetics. ${ }^{24}$

Proton pump inhibitors seem to be modestly superior to placebo in functional dyspepsia, but patients with dysmotility-like dyspepsia do not respond. ${ }^{13}$ In contrast, prokinetics have generally been found to be superior to placebo, although negative findings have also been published..$^{22}$

Erythromycin is a macrolide antibiotic with prokinetic properties, but its side effects and rapid tachyphylaxis limit its clinical utility. ${ }^{26}$ Visceral analgesics (e.g., fedotozine), antispas- 
modics (e.g., trimebutine), antinauseants (e.g., ondansetron), antidepressants (e.g., amitryptiline), and $H$ pylori eradication have also been tested in functional dyspepsia but are not of established benefit. ${ }^{262829}$ Little is known about the efficacy of behavioral therapy.

Choice of drug therapy

It seems logical to individualize drug therapy in functional dyspepsia. The committee believes that it is acceptable in general to consider an antisecretory or a prokinetic agent as first-line treatment for the patient with ulcer-like and dysmotility-like dyspepsia, respectively. Intermittent treatment courses (e.g., 2-4 weeks) may be considered when symptoms significantly impact on the patient's quality of life. In the rare patient whose symptoms are unremitting and incapacitating in the absence of medication, continuous therapy may be required. This approach needs appropriate testing in community trials.

There is evidence that symptoms in most patients with $H$ pylori positive functional dyspepsia do not improve with eradication of the organism. ${ }^{28} 29$ The physician faced with the problem of a patient with functional dyspepsia who wishes to prescribe eradication therapy should proceed to treatment only after explaining to the patient that there can be no confident expectation of symptomatic benefit and that there is a small risk of adverse reactions.

\section{B2. Aerophagia}

DEFINITION

Aerophagia is an unusual presenting complaint. It refers to a repetitive pattern of swallowing or ingesting air and belching. It is usually an unconscious act unrelated to meals, and is presumably a learned habit.

DIAGNOSTIC CRITERIA

At least 12 weeks, which need not be consecutive, in the preceding 12 months of:

(1) Air swallowing that is objectively observed; and

(2) Troublesome repetitive belching.

RATIONALE FOR CHANGES IN DIAGNOSTIC

CRITERIA

The committee concluded, based on strong clinical impressions, that aerophagia cannot be firmly diagnosed without observation of the occurrence of excessive air swallowing. As belching is a normal phenomenon, the committee concluded that the symptom must be troublesome to be clinically relevant. Bloating or other symptoms that are part of discomfort may temporarily benefit from belching in these patients. As visible abdominal distension is often part of the IBS rather than a gastroduodenal disorder, and as belching may not reduce distension, a requirement that belching relieves visible distension was considered unlikely to be of value.

CLINICAL EVALUATION

A positive diagnosis is based on a careful history and observation of air swallowing. In typical cases no investigation is required. Patients with GERD have increased air swallowing, but this is usually clearcut clinically. It is important to screen for psychiatric disease, including depression and anxiety.

\section{TREATMENT}

Explanation of the symptoms and reassurance are important. The habit can sometimes be unlearned by demonstrating chest expansion and air ingestion as the patient belches. Treatment of associated psychiatric disease or use of stress reduction techniques may be worth considering.

Dietary modification (avoiding sucking hard candies or chewing gum, eating slowly and encouraging small swallows at mealtime, and avoiding carbonated beverages) is often recommended, but has not been rigorously tested and is usually disappointing in practice.

Although tranquilizers may occasionally be useful in severe cases, ${ }^{30}$ drug therapy is not generally recommended. The value of psychologic interventions is essentially unknown.

\section{B3. Functional vomiting}

DEFINITION

Nausea is a subjective symptom that most people describe as a queasy, sick to the stomach sensation that may progress to the sense of a need to vomit. Vomiting is the forceful expulsion of gastric contents from the stomach. In functional vomiting, recurrent vomiting is the main presenting complaint and all known medical and psychiatric causes for the problem have been excluded.

DIAGNOSTIC CRITERIA

At least 12 weeks, which need not be consecutive, in the preceding 12 months of:

(1) Frequent episodes of vomiting, occurring on at least three separate days in a week; and

(2) Absence of criteria for an eating disorder, rumination, or major psychiatric disease according to DSM-IV; and

(3) Absence of self-induced and medication-induced vomiting; and

(4) Absence of abnormalities in the gut or central nervous system, and metabolic diseases to explain the recurrent vomiting.

A working definition of frequent episodes of vomiting adopted by the committee is at least one vomiting episode on three separate days in a week. This definition requires validation. The term psychogenic vomiting has no standard definition in the literature. The committee recommends that its use be abandoned in favor of functional vomiting as defined above.

\section{CLINICAL EVALUATION}

Functional vomiting is a rare condition in clinical practice. It should be distinguished from vomiting that occurs occasionally in patients with functional dyspepsia. The differential diagnosis of vomiting is extensive. Drugs should be excluded. It is important to rule out 
mechanical obstruction of the gastrointestinal tract and central nervous system disease. Initial relevant tests include an upper endoscopy and small bowel radiography. A metabolic screen is essential to exclude electrolyte and other abnormalities. A computed tomography or ultrasound scan of the abdomen may also be indicated to exclude other intra-abdominal disease depending on the clinical setting. If these tests are normal, then an assessment of gastric neuromuscular function (e.g., gastric emptying) is required. Gastroduodenal manometry is useful to exclude a myopathic or neuropathic process, and can help to identify a missed obstructive disorder of the intestine. The EGG may be helpful when combined with gastric emptying assessment to evaluate otherwise unexplained vomiting. ${ }^{31}$ Vomiting can occasionally be an atypical presentation of GERD which requires 24 hour esophageal $\mathrm{pH}$ testing to detect. ${ }^{32}$ Autonomic function testing, if abnormal, may point to a central nervous system process which may be confirmed by a magnetic resonance image of the brain. Psychiatric disease as the primary cause also needs to be excluded. Vomiting needs to be distinguished from rumination, where there is effortless regurgitation of undigested food during or after every meal; the patient spits out or reswallows the food, which is not sour or bitter. ${ }^{33}$ This syndrome is fully discussed in the chapter on the functional esophageal disorders. ${ }^{34}$

TREATMENT

Assessment of nutritional status is vital and appropriate intervention provided if there is a need. Antinauseants are worth a trial but are not of established value. Anecdotally, antidepressants can be of helpful in full doses. Psychosocial support is important. Behavioral and psychotherapy have not been adequately tested but may be considered.

1 Talley NJ, Colin-Jones D, Koch KL, et al. Functional dyspepsia. A classification with guidelines for diagnosis and management. Gastroenterol Int 1991;4:145-60.

2 Klauser AG, Schindlbeck NE, Muller-Lissner SA. Symptoms in gastro-esophageal reflux disease. Lancet 1990;335: 205-8.

3 Sonnenberg A, Delco F, El-Serag H. Empiric therapy versus Sonnenberg A, Delco F, El-Serag H. Empiric therapy versus
diagnostic tests in gastroesophageal reflux disease. A medidiagnostic tests in gastroesophageal reflux disease.

4 Talley NJ, Silverstein M, Agreus L, et al. AGA Technical Talley NJ, Silverstein M, Agreus L, et al. AGA Technical
review. Evaluation of dyspepsia. Gastroenterology 1998;114: review. Eval

5 Bytzer P, Hansen JM, Schaffalitzky de Muckadell OB, et al. Predicting endoscopic diagnosis in dyspeptic patients. The value of predictive score models. Scand $f$ Gastroenterol 1997;32:118-25.

6 Forbes GM, Glaser ME, Cullen DJ, et al. Duodenal ulcer treated with Helicobacter pylori eradication: seven-year follow-up. Lancet 1994;343:258-60.

7 Chiba N, Bernard L, O'Brien BJ, et al. A Canadian physician survey of dyspepsia management. Can $\mathcal{F}$ Gastroenterol 1998;12:83-90.
8 Colin-Jones DG, Bloom B, Bodemar G, et al. Management of dyspepsia. Report of a working party. Lancet 1988;i: of dysp

9 Drossman DA, Thompson GW, Talley NJ, et al. Identification of subgroups of functional gastrointestinal disorders. Gastroenterol Int 1990;3:159-72.

10 Heading RC. Definitions of dyspepsia. Scand f Gastroenterol 1991;26(suppl 182):1-6.

11 Agreus L, Talley NJ. Dyspepsia management in general practice. BMF 1997;315:1284-8.

12 Stanghellini V, Tosetti C, Paternico A, et al. Risk indicators of delayed gastric emptying of solids in patients with functional dyspepsia. Gastroenterology 1996;110:1036-42.

13 Tack J, Piessevaux H, Coulie B, et al. Role of impaired gastric accommodation to a meal in functional dyspepsia. Gastroenterology 1998;115:1586-7.

14 Talley NJ, Meineche-Schmidt V, Pare P, et al. Efficacy of omeprazole in functional dyspepsia. Double-blind, randomized placebo controlled trials (the Bond and Opera studies). Aliment Pharmacol Ther 1998;12:1055-65.

15 Hansen JM, Byzter P, Schaffalitzky de Muckadell OB. Placebo-controlled trial of cisapride and nizatidine in unselected patients with functional dyspepsia. Am $\mathcal{F}$ Gastroenterol 1998;93:368-74.

16 Small PK, Loudon MA, Waldron B, et al. Importance of reflux symptoms in functional dyspepsia. Gut 1995;36: 189-92.

17 Talley NJ, Zinsmeister AR, Schleck CD, et al. Dyspepsia and dyspepsia subgroups. A population based study. Gastroenterology 1992;102:1259-68.

18 Talley NJ, Boyce P, Jones M. Identification of distinct upper and lower gastrointestinal symptom groupings in the urban population. Gut 1998;42:690-5.

19 Malfertheiner P, Megraud F, O Morain C, et al. Current European concepts in the management of Helicobacter pylori infection-the Maastricht Consensus Report. The European Helicobacter pylori Study Group [EHPSG]. Eur f Gastroenterol Hepatol 1997;9:1-2.

20 McColl KE, el-Nujumi A, Murray L, et al. The Helicobacter pylori breath test: a surrogate marker for peptic ulcer disease in dyspeptic patients. Gut 1997;40:302-6.

21 Klauser AG, Voderholzer WA, Knesewitsch PA, et al. What is behind dyspepsia? Dig Dis Sci 1993;38:147-54.

22 Kellow JE, Cowan H, Shuter B, et al. Efficacy of cisapride therapy in functional dyspepsia. Aliment Pharmacol Ther 1995;9:153-60.

23 Jian R, Decrot F, Ruskone A, et al. Symptomatic, radionuclide and therapeutic assessment of chronic idiopathic dyspepsia. A double-blind placebo-controlled evaluation of cisapride. Dig Dis Sci 1989;34:657-64.

24 Finney JS, Kinnersley N, Hughes M, et al. Meta-analysis of antisecretory and gastrokinetic compounds in functional dyspepsia. F Clin Gastroenterol 1998;26:312-20.

25 Veldhuyzen van Zanten SJO, Cleary C, Talley NJ, et al. Drug treatment of functional dyspepsia. A systematic analysis of trial methodology with recommendations for the design of future trials. Report of an international working party. $\mathrm{Am}$ f Gastroenterol 1996;91:660-73.

26 Talley NJ. Functional dyspepsia-should treatment be targeted on disturbed physiology? Aliment Pharmacol Ther 1995;9:107-15.

27 Farup PG, Hovde O, Breder O. Are frequent short gastro-esophageal reflux episodes the cause of symptoms in patients with non-ulcer dyspepsia responding to treatment with ranitidine? Scand f Gastroenterol 1995;30:829-32.

28 Veldhuyzen van Zanten S, Talley NJ. Should antibiotic treatment of Helicobacter positive patients within nonulcer dyspepsia now be recommended? Eur $\mathcal{F}$ Gastroenterol Hepatol 1998;10:367-70.

29 Friedman LS. Helicobacter pylori and nonulcer dyspepsia. N Engl f Med 1998;339:1928-30.

30 Baume P, Tracey M, Dawson L. Efficacy of two minor tranquillizers in relieving symptoms of functional gastrointestinal distress. Aust NZ F Med 1975;5:503-6.

31 Koch KL. Dyspepsia of unknown origins. Pathophysiology, diagnosis and treatment. Dig Dis 1997;15:316-29.

32 Brzana RJ, Koch KL. Gastroesophageal reflux disease presenting with intractable nausea. Ann Intern Med 1997;126:704-7.

33 Malcolm A, Thumshirn MB, Camilleri M, et al. Rumination syndrome. Mayo Clin Proc 1997;72:646-52.

34 Clouse RE, Richter JE, Heading RC, et al. In: Drossman DA, Corazziari E, Talley NJ, et al, eds. Rome II: The functional gastrointestinal disorders. 2nd edn. McLean, VA: Degnon Associates, 2000. 